\title{
Singular Points in the Optical Center Distribution of P3P Solutions
}

\author{
Lihua Hu, ${ }^{1}$ Bo Wang, ${ }^{2}$ and Jifu Zhang' \\ ${ }^{1}$ School of Computer Science and Technology, Taiyuan University of Science and Technology, Taiyuan 030024, China \\ ${ }^{2}$ Institute of Automation, Chinese Academy of Sciences, Beijing 100190, China
}

Correspondence should be addressed to Lihua Hu; sxtyhlh@126.com

Received 27 April 2015; Revised 15 June 2015; Accepted 21 June 2015

Academic Editor: Erik Cuevas

Copyright (c) 2015 Lihua Hu et al. This is an open access article distributed under the Creative Commons Attribution License, which permits unrestricted use, distribution, and reproduction in any medium, provided the original work is properly cited.

Multisolution phenomenon is an important issue in P3P problem since, for many real applications, the question of how many solutions could possibly exist for a given P3P problem must at first be addressed before any real implementation. In this work we show that, given 3 control points, if the camera's optical center is close to one of the 3 toroids generated by rotating the circumcircle of the control point triangle around each one of its 3 sides, there is always an additional solution with its corresponding optical center lying in a small neighborhood of one of the control points, in addition to the original solution. In other words, there always exist at least two solutions for the P3P problem in such cases. Since, for all such additional solutions, their corresponding optical centers must lie in a small neighborhood of control points, the 3 control points constitute the singular points of the P3P solutions. The above result could act as some theoretical guide for P3P practitioners besides its academic value.

\section{Introduction}

The Perspective-3-Point Problem, or P3P problem, is a singleview based pose estimation method. It was first introduced by Grunert [1] in 1841 and popularized in computer vision community a century later by mainly Fischler and Bolles' work in 1981 [2]. Since it is the least number of points to have a finite number of solutions and no feature-matching across views is needed, it has been widely used in various fields [3-11], either for its minimal demand in restricted working environment, such as robotics and aeronautics, or for its computational efficiency acting as a minimum-set based solver repeatedly called in robust-statistics based iterative estimation framework, such as the well-known RANSAC-like framework, where the computational time increases exponentially with the cardinality of the minimum set; hence, the P3P problem is preferred due to its minimum requirement.

The P3P problem is defined as follows: Given the perspective projections of three control points with known coordinates in the world system and a calibrated camera, find the position and orientation of the camera in the world system. It is shown that the P3P problem could have 1, 2, 3, or at most 4 solutions depending on the configuration between the camera optical center and its 3 control points [12]. If the optical center and 3 control points happen to be concyclic, the problem becomes degenerated, and an infinitely large number of solutions could be possible.

Since, in many real applications, some basic questions must be answered before its real implementation (such as the following: Does it have a unique solution? If not, how many solutions could it have? Is the solution stable?), the multiple solution phenomenon in the $\mathrm{P} 3 \mathrm{P}$ problem has been a focus of investigation since its very inception in the literature. Traditionally the multisolution phenomenon in P3P problem is analyzed by at first transforming its 3 quadratic constraints into a quartic equation and then roots of this quartic equation are located to derive possible solutions. For example, Haralick et al. summarized 6 different transformation methods [12]. Gao et al. [13] gave a complete solution classification. Gao and Tang [14] also gave an analysis on the solutions distribution from the probabilistic point of view. Recently, Rieck [15-17] gave a systematic analysis on the multisolution phenomenon, in particular, on the cases where the optical center of the P3P problem is close to the danger cylinder. From the geometric point of view, Zhang and $\mathrm{Hu}$ [18] showed that when the optical center lies on the danger cylinder, the corresponding 
P3P problem must have 3 distinct solutions, and one must be a double solution. Lowe [3] and Zhang and $\mathrm{Hu}$ [19] showed that when the optical center lies on the three vertical planes perpendicular to the control point plane and going through one of the 3 altitudes of the control point triangle, the P3P problem must have a pair of side-sharing solutions and a pair of point-sharing solutions. (As shown in [19], a pair of side-sharing solutions refers to two such solutions that if their optical centers are superposed, they must share two control points or share a common side of the control point triangle. Similarly, a pair of point-sharing solutions refers to two such solutions that if their optical centers are superposed, they must share a single common control point.) Sun and Wang [20] gave an interpretation of the solution changes at the intersecting lines of the above three vertical planes with the danger cylinder. $\mathrm{Wu}$ and $\mathrm{Hu}$ [21] gave a thorough investigation on the degenerate cases in their "The PnP Problem Revisited" work. Recently, Wang et al. [22] show that the side-sharing pair of solutions is usually accompanied by a point-sharing pair of solutions, or the two kinds of pairs are often companion pairs. They also show [23] that if the optical center is outside of all the 6 toroids, each positive root of Grunert's quartic equation must correspond to a positive solution of the $\mathrm{P} 3 \mathrm{P}$ problem. Their work shows that the rootsolution-relationship is of one-to-one correspondence when the optical center is outside of all the 6 toroids but much more complicated when the optical center is inside of them.

In this work, we investigate the multisolution phenomenon in P3P problem for those cases where the camera optical center is very close to one of the 3 toroids. More specifically, based on Grunert's derivation [12], we show that when the optical center of a given $\mathrm{P} 3 \mathrm{P}$ problem is close to any one of the 3 toroids generated by rotating the circumcircle of the control point triangle around each of its 3 sides, in addition to its original solution, there is always an additional solution whose optical center always lies in a small neighborhood of control points. In other words, such a P3P problem always has at least two solutions. In addition, since, for all such additional solutions, their optical centers must be in a close neighborhood of control points, the 3 control points are singular points of the solutions' optical centers distribution.

\section{P3P Problem, 3 Toroids, and Grunert's Quartic Equation}

2.1. P3P Problem. As shown in Figure $1, A, B$, and $C$ are the three control points with known distance: $a=|B C|, b=|A C|$, and $c=|A B| ; O$ is the camera optical center. Since the camera (under the pinhole model) is calibrated, the three subtended angles $\alpha, \beta$, and $\gamma$ of the projection rays can be considered as known entities and then, by the Law of Cosines, the following 3 basic constraints on the three unknowns $s_{1}=|O A|, s_{2}=$ $|O B|$, and $s_{3}=|O C|$ in (1) must hold:

$$
\begin{gathered}
s_{1}^{2}+s_{2}^{2}-2 \cos \gamma s_{1} s_{2}=c^{2}, \\
s_{1}^{2}+s_{3}^{2}-2 \cos \beta s_{1} s_{3}=b^{2}, \\
s_{2}^{2}+s_{3}^{2}-2 \cos \alpha s_{2} s_{3}=a^{2} .
\end{gathered}
$$

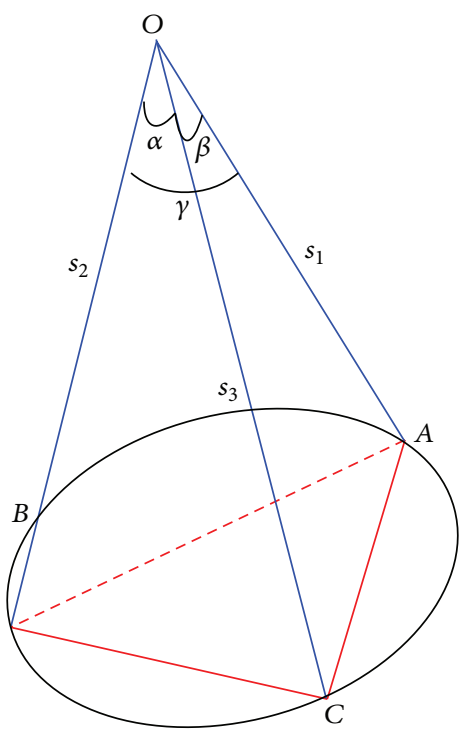

Figure 1: P3P problem definition: $A, B$, and $C$ are the 3 control points and $O$ is the camera optical center.

Hence, the P3P problem means to determine such positive triplets $\left(s_{1}, s_{2}, s_{3}\right)$ satisfying all the 3 basic constraints in (1). And the multisolution phenomenon refers to the existence of multiple such positive triplets $\left(s_{1}, s_{2}, s_{3}\right)$ all satisfying the 3 constraints in (1).

2.2. The 3 Toroids. As shown in Figure 1, by rotating the circumcircle of the triangle $A B C$ around one of its three sides by $2 \pi$ separately, 3 pairs of toroids (or 6 toroids) can be generated [24] as shown in Figure 2. For each toroid pair, one is generated by rotating the arc with the subtended angle $\angle A B C$ (or $\angle B A C$, or $\angle A C B$ ) and the other with its supplement angle $(\pi-\angle A B C)$ (or $(\pi-\angle B A C)$, or $(\pi-\angle A C B))$. In this work, the 3 toroids refer to the 3 ones generated only with the subtended angle $\angle A B C, \angle B A C$, and $\angle A C B$, denoted by Toroid_AC, Toroid $B C$, and Toroid_ $A B$, respectively. Hence, when the optical center lies on Toroid $A C$ (or Toroid $B C$, or Toroid_AB), the subtended angle $\beta$ (or $\alpha$, or $\gamma$ ) of the two projection rays $(O A, O C)$ (or $(O B, O C)$, or $(O A, O B))$, by definition, always has $\beta=\angle A B C$ (or $\alpha=\angle B A C$, or $\gamma=\angle A C B)$.

2.3. Grunert's Derivation. As shown in [12], by setting $u=$ $s_{2} / s_{1}$ and $v=s_{3} / s_{1}$, (1) can be transformed into a quartic equation in $v$ as

$$
A_{4} v^{4}+A_{3} v^{3}+A_{2} v^{2}+A_{1} v+A_{0}=0
$$

where

$$
\begin{aligned}
& A_{4}=\left(\frac{a^{2}-b^{2}-c^{2}}{b^{2}}\right)^{2}-\frac{4 c^{2}}{b^{2}} \cos ^{2} \alpha, \\
& A_{3}=4\left[\frac{a^{2}-c^{2}}{b^{2}}\left(\frac{b^{2}+c^{2}-a^{2}}{b^{2}}\right) \cos \beta\right.
\end{aligned}
$$




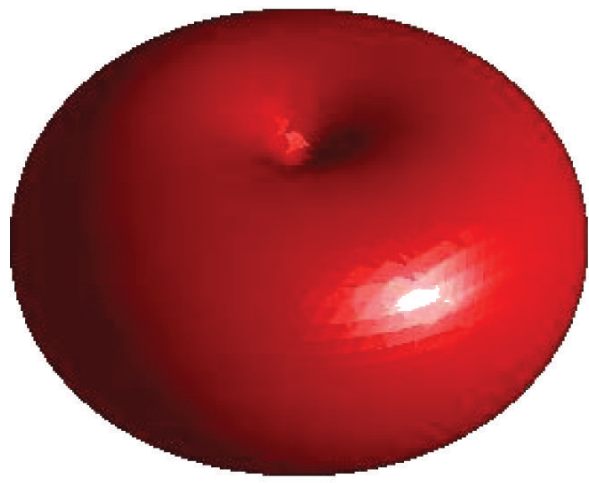

(a)

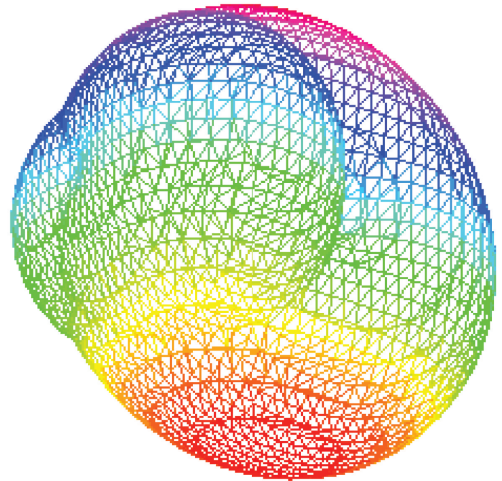

(b)

FIgURE 2: (a) A single toroid; (b) three toroids.

$$
\begin{aligned}
& \left.-\left(\frac{b^{2}-c^{2}-a^{2}}{b^{2}}\right) \cos \alpha \cos \gamma+2 \frac{c^{2}}{b^{2}} \cos ^{2} \alpha \cos \beta\right], \\
& A_{2}=2\left[\left(\frac{a^{2}-c^{2}}{b^{2}}\right)^{2}-1+2\left(\frac{a^{2}-c^{2}}{b^{2}}\right)^{2}\right. \\
& \cdot \cos ^{2} \beta+2\left(\frac{b^{2}-c^{2}}{b^{2}}\right) \cos ^{2} \alpha-4\left(\frac{a^{2}+c^{2}}{b^{2}}\right) \cos \alpha \\
& \left.\cdot \cos \beta \cos \gamma+2\left(\frac{b^{2}-a^{2}}{b^{2}}\right) \cos ^{2} \gamma\right] \text {, } \\
& A_{1}=4\left[-\left(\frac{a^{2}-c^{2}}{b^{2}}\right)\left(\frac{a^{2}+b^{2}-c^{2}}{b^{2}}\right) \cos \beta+2 \frac{a^{2}}{b^{2}}\right. \\
& \left.\cdot \cos ^{2} \gamma \cos \beta-\left(\frac{b^{2}-a^{2}-c^{2}}{b^{2}}\right) \cos \alpha \cos \gamma\right] \text {, } \\
& A_{0}=\left(\frac{a^{2}+b^{2}-c^{2}}{b^{2}}\right)^{2}-\frac{4 a^{2}}{b^{2}} \cos ^{2} \gamma \\
& s_{1}^{2}=\frac{b^{2}}{1+v^{2}-2 v \cos \beta} .
\end{aligned}
$$

$u$ can be determined from the following quadratic equation:

$$
u^{2}-2 u v \cos \alpha+\frac{b^{2}-a^{2}}{b^{2}} v^{2}+2 v \frac{a^{2}}{b^{2}} \cos \beta-\frac{a^{2}}{b^{2}}=0 .
$$

From (4), it can be seen that, given a positive root $v$ in (2), we can always obtain a positive $s_{1}$. From (5), if a positive $u$ can be obtained, then the positive triplet $\left(s_{1}, s_{2}=u s_{1}, s_{3}=v s_{1}\right)$ must be a positive solution of the P3P problem. Note that, given a positive root $v$ in (2), there could be $0,1,2$ positive roots of $u$ in (5). In other words, each positive $v$ could correspond to $0,1,2$ positive solutions of the $\mathrm{P} 3 \mathrm{P}$ problem. In [23], the authors showed that if the optical center is outside of all the 6 toroids, each positive root must correspond to a unique positive solution of the $\mathrm{P} 3 \mathrm{P}$ problem. In the next section, we show that when the optical center is close to one of the 3 toroids in Section 2.2, the corresponding P3P problem must have at least two different positive solutions, the one whose optical center is at the original position and the other whose optical center is in a small neighborhood around one of the 3 control points. Here "two different solutions" refer to "the two solutions with the same 3 control points, but their optical centers lie at a different position."

\section{Solution Singularity in P3P Problem}

We have the following main result.

Proposition 1. As shown in Figures 1 and 2, if the optical center $O$ is close to Toroid_AB, then except for some special curves, there is always another optical center $O^{\prime}$ in a small neighborhood of control point $C$ such that $\left\{O^{\prime},(A, B, C)\right\}$ is another positive solution of the $P 3 P$ problem in addition to its original solution $\{O,(A, B, C)\}$.

Proof. If the optical center $O$ lies on the Toroid_AB, $\gamma=$ $\angle A C B$. Hence, $A_{0}=0$, and $v=0$ is a root of the quartic equation in (2).

Suppose the optical center $O$ is not on the Toroid $A B$, but very close to it; then the three subtended angles from $O$ to the three control points $A, B$, and $C$ are as follows: $\alpha, \beta, \gamma=$ $\angle A C B+\Delta \gamma$, where $\Delta \gamma$ is quite small since $O$ is very close to Toroid $\_A B$. In fact, $\Delta \gamma$ could be infinitesimally approaching 0 if the optical center is infinitesimally close to Toroid $\_A B$.

Since $\Delta \gamma$ is very small, all the 5 coefficients $A_{i}, i=$ $0,1,2,3,4$, can be approximated by its linear term at $\alpha, \beta$, $\gamma=\angle A C B$. In other words, we can keep $\alpha, \beta$ unchanged, but only $\gamma$ is subject to a small change by $\Delta \gamma$ at $\gamma=\angle A C B$.

When $\gamma=\angle A C B, v=0$ is a root of the quartic equation in (2). When $\gamma=\angle A C B+\Delta \gamma$, there should be a root $\Delta v$ of the linearized equation of (2) at $\alpha, \beta, \gamma=\angle A C B$. Since (2) is a quartic equation, we need only to consider the last two terms: that is,

$$
\left(A_{1}+\Delta A_{1}\right)(\Delta v)+A_{0}+\Delta A_{0}=0 .
$$


If $A_{1} \neq 0$ when $\gamma=\angle A C B$, and since $A_{0}=0$ when $\gamma=$ $\angle A C B$, then if $\triangle A_{0} \neq 0$, we have

$$
\Delta v=-\frac{\Delta A_{0}}{A_{1}} \neq 0 .
$$

Let us consider the condition of $A_{1}=0$ and that of $\Delta A_{0}=0$ when $\gamma=\angle A C B$.

When $\gamma=\angle A C B$, if $A_{1}=0$, we have

$$
\begin{gathered}
-\left(\frac{a^{2}-c^{2}}{b^{2}}\right)\left(\frac{a^{2}+b^{2}-c^{2}}{b^{2}}\right) \cos \beta+2 \frac{a^{2}}{b^{2}} \cos ^{2} \angle A C B \\
\cdot \cos \beta-\left(\frac{b^{2}-a^{2}-c^{2}}{b^{2}}\right) \cos \alpha \cos \angle A C B=0 .
\end{gathered}
$$

Since $\cos \angle A C B=\left(a^{2}+b^{2}-c^{2}\right) / 2 a b$, substituting it into the above equation and by some manipulations, the above equation can be simplified as

$$
\cos \angle A C B(\cos \beta \cos \angle B A C+\cos \alpha \cos \angle A B C)=0 .
$$

$\cos \angle A C B=0$ means $\angle A C B=\pi / 2$, or the control point triangle is a right triangle. In this case, the Toroid $\_A B$ degenerates into a sphere.

If $\angle A C B \neq \pi / 2, A_{1}=0$ indicates that the 3 subtended angles $(\alpha, \beta, \gamma=\angle A C B+\triangle \gamma)$ of the optical center $O$ with the three control points $A, B$, and $C$ must satisfy the following relation:

$$
\frac{\cos \angle B A C}{\cos \alpha}=-\frac{\cos \angle A B C}{\cos \beta} .
$$

Since $\Delta A_{0}$ can be expressed as

$$
\Delta A_{0}=\frac{4 a^{2}}{b^{2}} \sin (2 \angle A C B) \Delta \gamma,
$$

then if $\angle A C B \neq \pi / 2, \triangle A_{0} \neq 0$.

In conclusion, if $\angle A C B \neq \pi / 2$, and if $\cos \angle B A C / \cos \alpha \neq$ - $\cos \angle A B C / \cos \beta, \Delta v=-\Delta A_{0} / A_{1}$ always exists, and we can always make $\Delta v=-\Delta A_{0} / A_{1}>0$ by choosing either $\Delta \gamma>0$ or $\Delta \gamma<0$. Geometrically, $\Delta \gamma>0$ means the optical center $O$ is inside of Toroid $A B$, and $\Delta \gamma<0$ means the optical center $O$ is outside of Toroid $A B$.

Since $\Delta v \rightarrow 0$ when $\Delta \gamma \rightarrow 0$, from (5), $u \approx$ $a / b$. From $\Delta v>0, u \approx a / b>0$, a positive triplet $\left(s_{1}, s_{2}, s_{3}\right)$ can be obtained, which must be another solution of the $\mathrm{P} 3 \mathrm{P}$ problem. Since $\Delta v$ is small, $s_{3}=$ $\Delta v \times s_{1}=\Delta v \sqrt{b^{2} /\left(1+(\Delta v)^{2}-2(\Delta v) \cos \beta\right)} \approx b \Delta v=$ $-\left(\left(4 a^{2} / b\right) \sin (2 \angle A C B) / A_{1}\right) \Delta \gamma$. Hence, when $\Delta \gamma \rightarrow 0, s_{3} \rightarrow$ 0 , it indicates that the corresponding optical center $O^{\prime}$ of this additional solution must be close to the control point $C$. In other words, $O^{\prime}$ is in a close neighborhood of control point $C$. Hence, the proposition is proved.

Remark 2. When the optical center $O$ is close to Toroid_AC (or Toroid $B C$ ), by setting the variables $v=s_{2} / s_{3}, u=s_{1} / s_{3}$ (or $v=s_{1} / s_{2}, u=s_{3} / s_{2}$ ), we can similarly prove that there exists additionally a positive solution whose corresponding optical center $O^{\prime}$ lies in a close neighborhood of control point $B$ (or $A$ ).
Remark 3. When $\triangle A B C$ is a right angle, say $\angle A C B=\pi / 2$, Toroid_AB becomes a sphere, and this is quite a special case. As shown by Zhang and $\mathrm{Hu}$ [18] and Sun and Wang [20], the pair of side-sharing solutions degenerates into a double solution in this case.

Remark 4. In fact, "the closeness of neighborhood" in the above discussions can be explicitly quantified as $s_{3} \approx$ $-\left(4 a^{2} \sin (2 \angle A C B) / A_{1} b\right) \Delta \gamma$, or a linear relationship between $s_{3}$ (the neighborhood size) and $\Delta \gamma$ (the closeness magnitude of the optical center $O$ to the toroid).

Remark 5. From the above discussion, we can see that if the optical center is close to one of the 3 toroids (inside or outside of the toroid), except for some special curves, there is always a P3P positive solution whose optical center is in a close neighborhood of one of the three control points. This indicates that the 3 control points are singular points of the optical centers distribution of the P3P solutions.

\section{Computer Simulation}

Although the correctness of our proposition lies in its proof, we nevertheless also verify it by computer simulations. Our simulation procedure is as follows.

Generate 3 noncollinear control points $A, B$, and $C$.

Repeat (1)-(4).

(1) Choose at random an optical center $O$ close to one of the 3 toroids.

(2) From $\{O,(A, B, C)\}$, compute the three quadratic constraints in (1) and then transform them into a quartic equation in (2).

(3) Compute the roots of (2) to determine a small root $\Delta v$.

(4) Compute the P3P solution corresponding to $\Delta v$ as shown in Section 3.

We generate about 10,000 triplets of control points, and for each triplet, about 10,000 different optical centers are simulated. All the simulations confirm our general theoretical conclusion; that is, the optical centers of all the additional P3P solutions are in a close neighborhood of control points. Figure 3 is an example of the resulting optical center distributions around the control point $A$ (for the visualization clarity purpose, only 100 points are shown). In Figure 3(a), the blue points are the optical centers near the Toroid_BC, the green point is the control point $B$, the pink point is the control point $C$, and the red points are the optical centers of those additional corresponding $\mathrm{P} 3 \mathrm{P}$ solutions, which are all near the control point $A$. Figure 3(b) is the enlarged part near the control point $A$, and it shows that these optical centers are in a small neighborhood of $A$. Each blue point in Figure 3(a) has a corresponding red point in Figure 3(b). In other words, a blue point and the 3 control points constitute a $\mathrm{P} 3 \mathrm{P}$ problem whose optical center (the blue point) is close to Toroid_BC, and this $\mathrm{P} 3 \mathrm{P}$ problem has another solution (the corresponding red point and the 3 control points) whose optical center (the red point) is close to the control point $A$. 


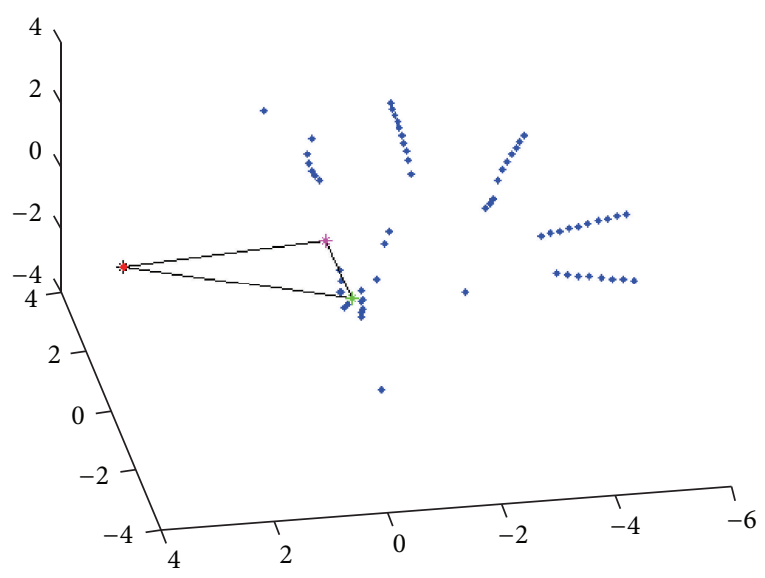

(a)

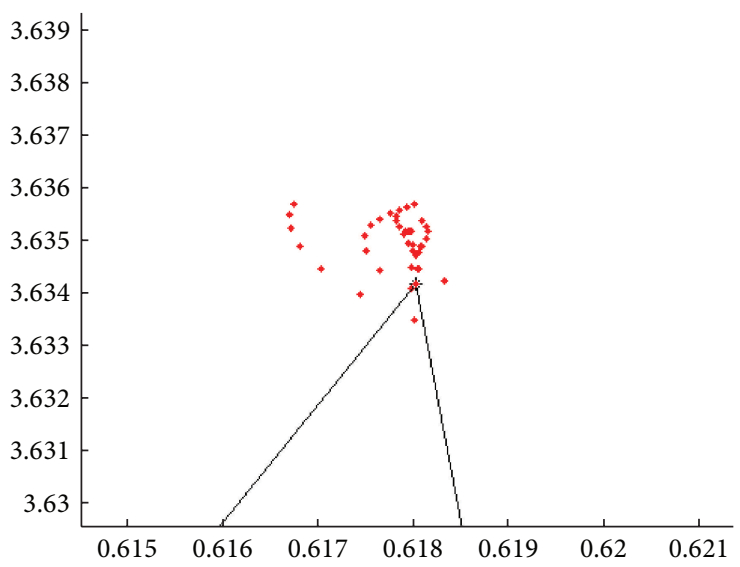

(b)

FIGURE 3: An example of the resulting optical center distributions around the control point $A$.

\section{Conclusion}

In this work, we find that, given 3 control points, if the camera optical center is close to any one of the 3 toroids, except for some special curves, there is always an additional positive solution for this P3P problem whose corresponding optical center always lies in a close neighborhood of one of the 3 control points. Hence, the 3 control points are singular points of the optical centers distribution of the P3P solutions. In other words, some singularity exists around the 3 control points for the P3P problem solutions.

Our result provides some new insights into the nature of multisolution phenomenon; in addition to its academic value, the result could also provide some theoretical guidance for $\mathrm{P} 3 \mathrm{P}$ practitioners to properly arrange their control points to avoid unstable solutions for the P3P based applications.

\section{Conflict of Interests}

The authors declare that there is no conflict of interests regarding the publication of this paper.

\section{Acknowledgment}

This work was supported by National Natural Science Foundation of China (NSFC) under Grant nos. 61402316 and 61403373.

\section{References}

[1] J.A. Grunert, "Das pothenotische problem in erweiterter gestalt nebst über seine anwendungen in der geodäsie," Grunerts Archiv für Mathematik und Physik, vol. 1, pp. 238-248, 1841.

[2] M. A. Fischler and R. C. Bolles, "Random sample consensus: a paradigm for model fitting with applications to image analysis and automated cartography," Communications of the Association for Computing Machinery, vol. 24, no. 6, pp. 381-395, 1981.

[3] D. G. Lowe, "Fitting parameterized three-dimensional models to images," IEEE Transactions on Pattern Analysis and Machine Intelligence, vol. 13, no. 5, pp. 441-450, 1991.
[4] V. Lepetit and P. Fua, "Monocular model-based 3D tracking of rigid objects: a survey," Foundations and Trends in Computer Graphics and Vision, vol. 1, no. 1, pp. 1-89, 2005.

[5] C. McGlove, E. Mikhail, and J. Bethel, Manual of Photogrametry, American Society for Photogrammetry and Romote Sensing, 5th edition, 2004.

[6] D. Nistér and H. Stewénius, "A minimal solution to the generalised 3-point pose problem," Journal of Mathematical Imaging and Vision, vol. 27, no. 1, pp. 67-79, 2007.

[7] J. Qingxuan, Z. Ping, and S. Hanxu, "The study of positioning with igh-precision by single camera based on P3P algorithm," in Proceedings of the IEEE International Conference on Industrial Informatics (INDIN' 06), pp. 1385-1388, August 2006.

[8] L. Quan and Z. Lan, "Linear N-point camera pose determination," IEEE Transactions on Pattern Analysis and Machine Intelligence, vol. 21, no. 8, pp. 774-780, 1999.

[9] J. Tang, W. Chen, and J. Wang, "A study on the P3P problem," in Advanced Intelligent Computing Theories and Applications. With Aspects of Theoretical and Methodological Issues, vol. 5226 of Lecture Notes in Computer Science, pp. 422-429, Springer, Berlin, Germany, 2008.

[10] J. Tang and N. Liu, "The unique solution for P3P problem," in Proceedings of the 2009 ACM Symposium on Applied Computing (SAC '09), pp. 1138-1139, ACM, New York, NY, USA, 2009.

[11] W. J. Wolfe, "A Special Case Solution to the Perspective 3-Point Problem," http://faculty.csuci.edu/william.wolfe/csuci/articles/ perspectiveview/new_p3p_article/wolfe_p3p_special_case.pdf.

[12] B. M. Haralick, C.-N. Lee, K. Ottenberg, and M. Nölle, "Review and analysis of solutions of the three point perspective pose estimation problem," International Journal of Computer Vision, vol. 13, no. 3, pp. 331-356, 1994.

[13] X.-S. Gao, X.-R. Hou, J. Tang, and H.-F. Cheng, "Complete solution classification for the perspective-three-point problem," IEEE Transactions on Pattern Analysis and Machine Intelligence, vol. 25, no. 8, pp. 930-943, 2003.

[14] X.-S. Gao and J.-L. Tang, "On the probability of the number of solutions for the P4P Problem," Journal of Mathematical Imaging and Vision, vol. 25, no. 1, pp. 79-86, 2006.

[15] M. Q. Rieck, "An algorithm for finding repeated solutions to the general perspective three-point pose problem," Journal of 
Mathematical Imaging and Vision, vol. 42, no. 1, pp. 92-100, 2012.

[16] M. Q. Rieck, "Solving the three-point camera pose problem in the vicinity of the danger cylinder," in Proceedings of the International Conference on Computer Vision Theory and Applications (VISAPP '12), vol. 2, pp. 335-340, February 2012.

[17] M. Q. Rieck, "A fundamentally new view of the perspective three-point pose problem," Journal of Mathematical Imaging and Vision, vol. 48, no. 3, pp. 499-516, 2014.

[18] C.-X. Zhang and Z.-Y. Hu, "Why is the danger cylinder dangerous in the P3P Problem?" Acta Automatica Sinica, vol. 32, no. 4, pp. 504-511, 2006.

[19] C.-X. Zhang and Z.-Y. Hu, "A general sufficient condition of four positive solutions of the P3P Problem," Journal of Computer Science and Technology, vol. 20, no. 6, pp. 836-842, 2005.

[20] F.-M. Sun and B. Wang, "A note on the roots distribution and stability of the PnP Problem," Acta Automatica Sinica, vol. 36, no. 9, pp. 1213-1219, 2010.

[21] Y.-H. Wu and Z.-Y. Hu, "PnP problem revisited," Journal of Mathematical Imaging and Vision, vol. 24, no. 1, pp. 131-141, 2006.

[22] B. Wang, C.-X. Zhang, and Z.-Y. Hu, "Geometric interpretation of the multi-solution phenomenon in the P3P problem," IEEE Transactions on Pattern Analysis and Machine Intelligence. Under revision.

[23] B. Wang, C.-X. Zhang, and Z.-Y. Hu, “The relationship between solutions of the $\mathrm{P} 3 \mathrm{P}$ problem and roots of its resulting quartic equation,” Tech. Rep. TR-CASIA-NLPR-RV-2015-03-07, 2015.

[24] W. J. Wolfe, D. Mathis, C. W. Sklair, and M. Magee, "The perspective view of three points," IEEE Transactions on Pattern Analysis and Machine Intelligence, vol. 13, no. 1, pp. 66-73, 1991. 


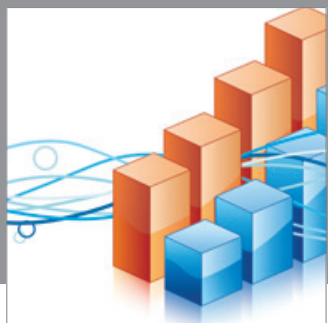

Advances in

Operations Research

mansans

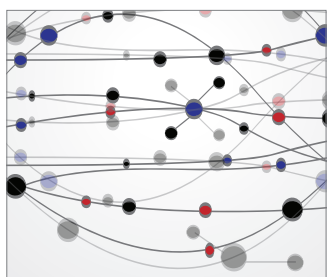

The Scientific World Journal
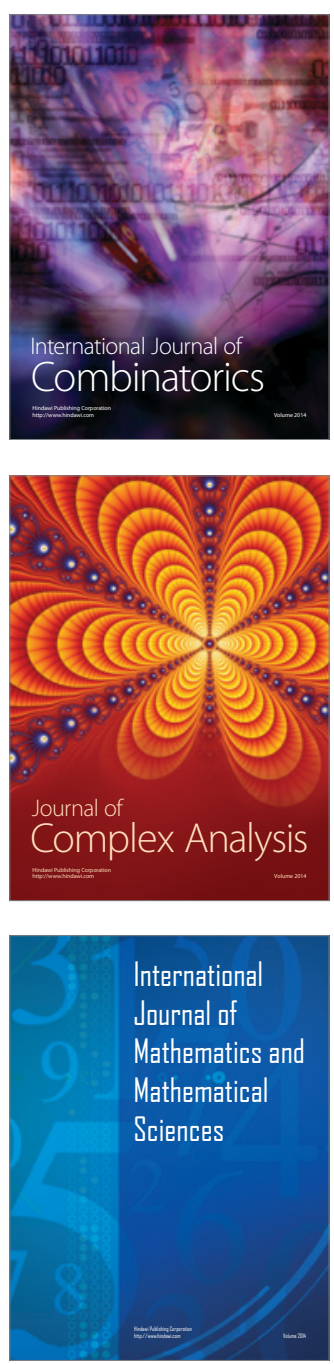
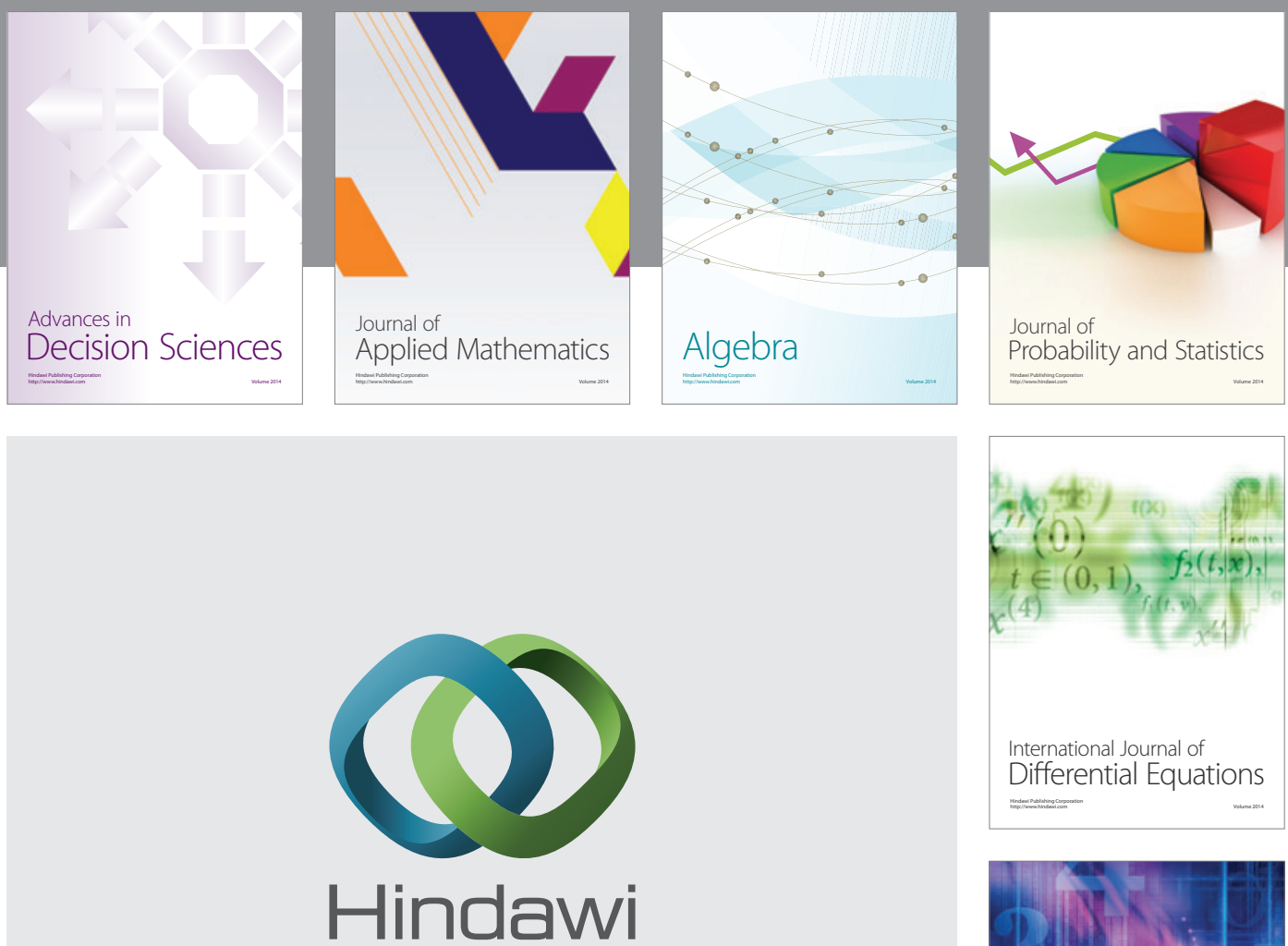

Submit your manuscripts at http://www.hindawi.com
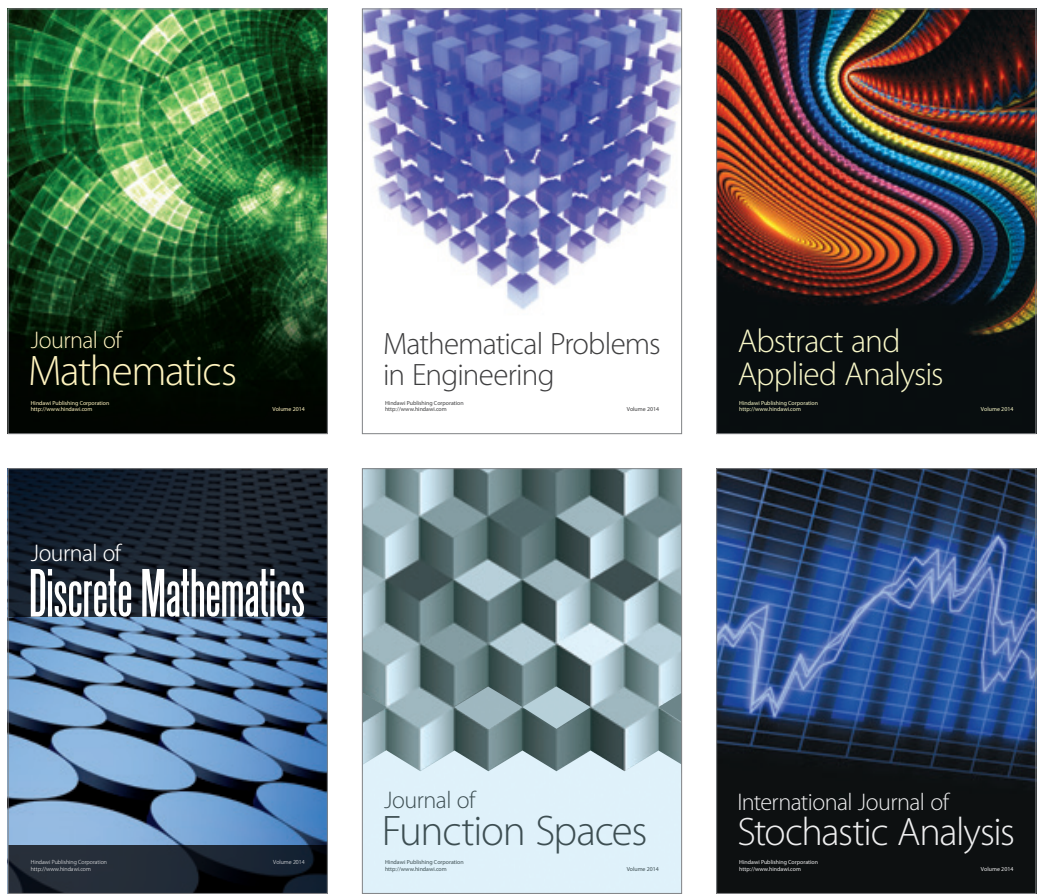

Journal of

Function Spaces

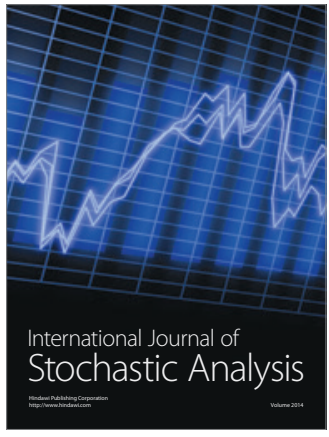

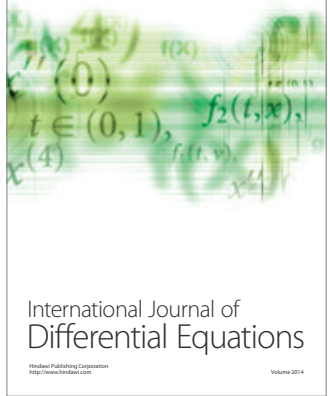
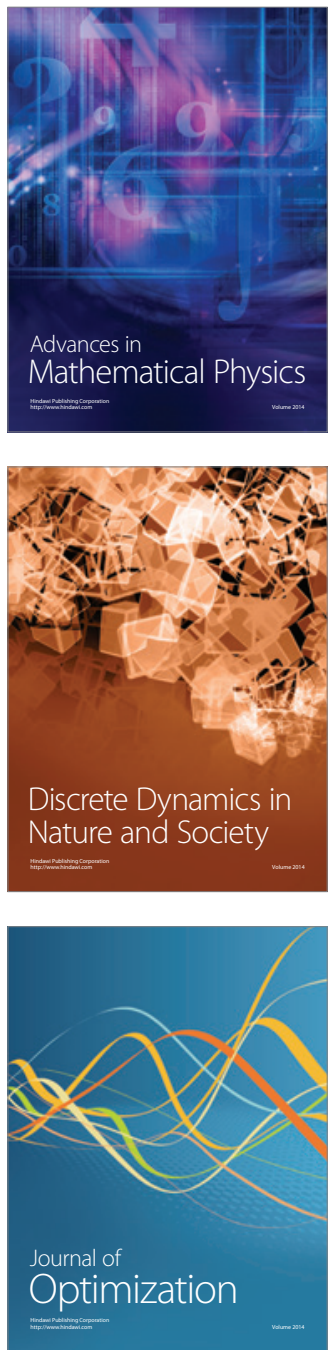